\title{
PROCESS AND SYSTEMS Precision reimbursement for precision medicine: the need for patient-level decisions between payers, providers and pharmaceutical companies
}

\author{
Authors: Sanjay Budhdeo, ${ }^{\mathrm{A}}$ Michael Ruhl, ${ }^{\mathrm{B}}$ Paul M Agapow, ${ }^{\mathrm{C}}$ Nikhil Sharma ${ }^{\mathrm{D}}$ and Parker Moss ${ }^{\mathrm{E}}$
}

\begin{abstract}
Healthcare costs have been dramatically rising in developed economies worldwide. A key driver of cost increases has been high-cost drugs. The current model of reimbursement is not configured for drugs with uncertain outcomes. Future reimbursement will require better allocation of available healthcare system funds. Technological advancements have opened the door to a new type of outcomes-based reimbursement, enabling value exchange between payers and pharmaceutical companies, which we term precision reimbursement. Precision reimbursement extends beyond value-based contracts, with decisions at individual rather than aggregate level. For precision reimbursement to be adopted, there are data, computation and infrastructure requirements. All stakeholders benefit in moving to precision reimbursement for optimal resource allocation, risk sharing and, ultimately, improved outcomes. There are implementation challenges including cost, change management, information governance and development of surrogate markers. The overarching trend in medicine is toward personalised interventions, with precision reimbursement as the logical consequence.
\end{abstract}

KEYWORDS: precision medicine, reimbursement, value-based healthcare, stratified medicine

DOI: $10.7861 /$ fhj.2021-0066

The case for reimbursement innovation

The current reimbursement model is unsustainable

Healthcare costs have been rising above the rate of inflation in developed economies internationally. One significant driver of cost increases has been new drugs. ${ }^{1}$ Scientific breakthroughs (including

Authors: ${ }^{A}$ Wellcome clinical training fellow, University College London, UK and National Hospital for Neurology and Neurosurgery, London, UK; ${ }^{\mathrm{B}}$ managing director and partner, Boston Consulting Group, Dusseldorf, Germany; ${ }^{C}$ data science director, AstraZeneca, Cambridge, UK; ${ }^{D}$ honorary consultant neurologist, University College London, London, UK and National Hospital for Neurology and Neurosurgery, London, UK; Enon-executive board member, Cancer Research UK, UK monoclonal antibodies, and cell and gene therapies) have led to a completely new health economic conundrum for commissioners and governments. These very high-priced therapeutics are potentially curative and so, in the long term, have direct and indirect economic benefits for the health system, the workforce and the country. Examples of these include Kymriah and Yescarta (chimeric antigen receptor T-cell therapies for haematologic malignancies approved by the US Food and Drug Administration in 2017), and Zolgensma (an adeno-associated virus gene therapy for spinal muscular atrophy). ${ }^{2-5}$ The current model of reimbursement is not configured for these high price point drugs, especially those with uncertain outcomes. Future reimbursement will require better allocation of available healthcare system funds to the most effective uses. Although the exact shape of the future reimbursement model is not yet clear, it will likely be anchored on an outcomes-based logic. We propose a new model, where outcomes are predicted and monitored at the patient, rather than population, level in order to tailor reimbursement to individual patient outcomes.

Previous attempts to move to outcomes-based reimbursement have had limited success

The classical outcomes-based reimbursement model, based on retrospective assessment of value using real-world evidence, is routinely applied to structure the way that payers and providers work together and exchange value, both in the Centers for Medicare \& Medicaid Services and the largest private American payers (such us United Healthcare). ${ }^{6}$ However, this classical model cannot easily be applied to structure the way that payers and pharmaceutical companies work together and exchange value. Pharmaceutical companies have been reluctant to enter into these agreements because of a lack of the control over care. Payers have concerns over the complexity of the required monitoring and other implementation logistics.

Like value-based healthcare (VBH) contracts, precision reimbursement focuses on payment for outcomes. The key difference is the granularity of data taken into account for reimbursement, with decisions at individual rather than aggregate levels.

Precision reimbursement is now possible at scale

Technological advancements have opened the door to a new type of outcomes-based reimbursement that is a better fit for the 
specific requirements of the value exchange between payers and pharmaceutical companies. This new model is prospective and anchored on patient-level predictions. It would work in four ways.

> Biopharmaceutical companies, in collaboration with key providers and/or payers, apply machine learning $(\mathrm{ML})$ to large real-world data (RWD) to identify clinical features that define a high-value patient (ie a patient at high event or disease progression risk, or at high failure risk on current standard-ofcare) and build out an ML-powered prediction model around those features to enable better clinical decision making.

> Pharmaceutical companies and payers collaboratively apply this prediction model to the population of interest to collaboratively model value in the payer data and align on a data-driven reimbursement level to fairly reflect value.

> The prediction model is made available to providers so that they can enhance their decision making around the high-value patients.

> Reimbursement for these high-value patients is automatically triggered when confirmed by the prediction model.

This design overcomes the hurdles that have made adoption of outcomes-based reimbursement so difficult in payer-pharmaceutical company interactions as it requires no pharmaceutical company control over care delivery and is logistically easy to implement.

The technological enablers for this approach include advancements in basic and applied ML, increasing adoption of data structures and ontologies (such as Fast Healthcare Interoperability Resources (FHIR) and Systematized Nomenclature of Medicine Clinical Terms (SNOMED-CT)), better processing through AI-adapted graphic processing units, and better access to data through data warehousing and federated learning. ${ }^{7-10}$ There are increasing examples of areas where $\mathrm{ML}$ has been used to generate patient-level outcome predictions, including in macular degeneration, multiple cancers and rare diseases. ${ }^{11-14}$

\section{ML-based precision reimbursement in combination with RWD-based real-time monitoring}

Three requirements for precision reimbursement to be adopted

For precision reimbursement to be adopted, there are data, computation and infrastructure requirements. ML relies upon having adequate volumes of relevant data. Suitable sources of data of sufficient volume will have to be cultivated; there are promising efforts in this direction. ${ }^{15,16}$ Computational resources for routinely and reproducibly building models must be made available. At the time of writing, many cloud providers are introducing such facilities and there is an increasing number of platforms for routine building and deployment of ML models. Procedures and infrastructure must be built that will allow for suitable governance and privacy while, at the same time, allowing full transparency into model construction and operation. This may be the most challenging requirement of the three but, if it can be mastered, we will have built a healthcare system that can learn from and respond to its patients.

\section{Precision reimbursement benefits all stakeholders}

Currently, most payers and providers have adopted volume-based pricing. However, high-priced drugs like checkpoint inhibitors only work for patients with specific biomarkers, and are typically priced significantly above the quality-adjusted life year limits of most developed countries and the price thresholds of many private payers. ${ }^{17}$ Value-based pricing is part of the solution to convince payers to invest by shifting some of the risk to the pharmaceutical companies. Payers will come under increasing pressure to provide these high-cost, highly efficacious drugs for patients. To do so, they need to shift some of the effectiveness risk back to the pharmaceutical companies, such that reimbursement is dependent on the response to treatment. In the long run, payers will be able to make better actuarial underwriting decisions because they will have access to both response to treatment predictions and outcome data, and we may see this VBH approach expanded beyond the high-priced drug segment. Response to treatment may be partly determined by biomarkers that are assessed through companion diagnostics, but quality of care and adherence to protocol also plays an important role. However, pharmaceutical companies do not have end-to-end control over pathway adherence. A deeper role in care delivery by pharmaceutical companies would be an expensive and operationally challenging prospect.

Providers should benefit from VBH contracts, which would embed the use of patient selection tools through companion diagnostics, with more granular protocols that lead to a high response to treatment across stratified patient populations. RWD from provider-generated data will become an increasingly important and commercially valuable asset for hospitals that manage their data assets effectively and ethically, which provides an incentive for hospitals to engage in value-based pricing and to invest in data quality.

Patients will be the greatest beneficiary of precision reimbursement, as the response to treatment models can be used to tailor treatment choice for each individual (Fig 1).

\section{Current challenges to implement precision reimbursement}

\section{Investing in change}

The technical enablers described earlier are all challenged by balance-sheet constraints of healthcare institutions, particularly in socialised health economies and also by the very challenging

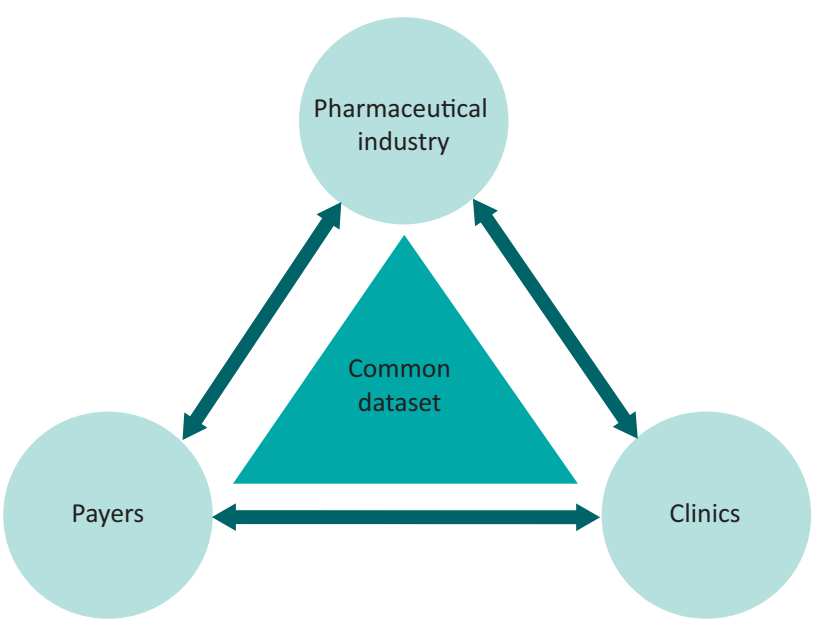

Fig 1. The three-way relationship for personalised, response to treatment, machine learning models. 
change management environment in healthcare. Behaviour change in the medical community is hard-won.

There are particular challenges in engaging payers in this model. For payers, precision reimbursement will require more sophisticated models of customer segmentation and longitudinal data management.

Engagement from governments and regulators is vital. Governments can ensure that legal frameworks are in place to put in guarantees for precision reimbursement, and regulators can enforce them. Governments and regulators can act as advocates for a new system at a national and international level, harmonising regulations and agreeing common standards.

\section{The information governance challenge}

There may be difficulties in sharing patient data across borders, due to the nature of data privacy laws such as General Data Protection Regulation and Health Insurance Portability and Accountability Act. There are three potential solutions. Firstly, federated learning would mitigate this challenge by leaving data in situ, on local servers at provider organisations, meaning that models can be developed while data can continue to be controlled by hospitals. Secondly, data warehousing with transfer learning would enable ML models to be refined across national borders without the need for data transfer. Lastly, developing international regulatory agreements would enable data from patients from different countries to be centralised in a single data warehouse. Any organisation that produces response to treatment models must be legally recognised as arbiters of pricing decisions in countries where this marketplace is implemented. ${ }^{10,18}$

\section{Timeline challenge}

Developing surrogate measures for long-term outcomes will be key in calibrating reimbursement accuracy and timing to make this vision a reality. Surrogate endpoints generation with ML will require prospective validation to ensure buy-in and fairness to all sides. In order to accelerate the design of next generation surrogate outcome measures, there will need to be a combination of novel, multi-modal data sources and deployment of advanced analytics including ML.

\section{Conclusion}

The overarching trend in medicine is toward personalised interventions, with precision reimbursement as the logical consequence. ML has the opportunity to ensure optimal utilisation of healthcare spending and fair valuation of treatment. At a time when the current model of reimbursement is under considerable strain, our proposal de-risks high-priced drugs for payers, creates viable markets for pharmaceutical companies and enables providers to offer the most innovative treatment options. A fair and transparent broker would have access to data and would use advanced predictive modelling techniques to span all use cases outlined; this could be a government agency or an independent company. Our proposal demonstrates how data can improve healthcare outcomes.

\section{Conflicts of interest}

This article represents the personal views of the authors only and does not reflect the views held by their affiliated institutions.
Sanjay Budhdeo owns stock in Owkin. Sanjay Budhdeo and Parker Moss were formerly employed by Owkin; Owkin is a company that uses federated learning for clinical development projects in collaboration with clinical and research institutions, serving biopharmaceutical companies. Michael Ruhl is a managing director and partner at Boston Consulting Group (BCG); BCG advises biopharmaceutical companies, payers and providers internationally. Paul M Agapow is an employee of AstraZeneca, a biopharmaceutical company.

\section{Funding}

Nikhil Sharma receives funding from NIHR University College London Hospitals Biomedical Research Centre and the Reta Lila Weston Trust. Sanjay Budhdeo is funded by Wellcome. This article did not receive any specific grant funding or support.

\section{References}

1 DiMasi JA, Grabowski HG, Hansen RW. Innovation in the pharmaceutical industry: New estimates of R\&D costs. Journal of Health Economics 2016;47:20-33.

2 Novartis Pharmaceuticals Corporation. KYMRIAH (tisagenlecleucel). US Food and Drug Administration, 2021. www.fda.gov/ vaccines-blood-biologics/cellular-gene-therapy-products/kymriahtisagenlecleucel

3 Kite Pharma. YESCARTA (axicabtagene ciloleucel). US Food and Drug Administration, 2021. www.fda.gov/vaccines-blood-biologics/ cellular-gene-therapy-products/yescarta-axicabtagene-ciloleucel

4 Harvey RD. New drug updates in hematologic malignancies: CAR-T, targeted therapeutics, and other agents. J Adv Pract Oncol 2018;9:282-6.

5 AveXis. ZOLGENSMA. US Food and Drug Administration, 2021. www.fda.gov/vaccines-blood-biologics/zolgensma

6 Squitieri L, Bozic KJ, Pusic AL. The role of patient-reported outcome measures in value-based payment reform. Value in Health 2017;20:834-6.

7 Topol EJ. High-performance medicine: the convergence of human and artificial intelligence. Nat Med 2019;25:44-56.

8 Benson T, Grieve G. Principles of FHIR. In: Benson T, Grieve G (eds). Principles of Health Interoperability: Health Information Technology Standards. Springer, 2016:329-48.

9 Benson T, Grieve G. SNOMED CT. In: Benson T, Grieve G (eds). Principles of Health Interoperability: Health Information Technology Standards. Springer, 2016:155-72.

10 Rieke N, Hancox J, Li W et al. The future of digital health with federated learning. NPJ Digit Med 2020;3:119.

11 Yim J, Chopra R, Spitz T et al. Predicting conversion to wet age related macular degeneration using deep learning. Nat Med 2020;26:892-9.

12 Huang C, Clayton EA, Matyunina LV et al. Machine learning predicts individual cancer patient responses to therapeutic drugs with high accuracy. Sci Rep 2018;8:1-8.

13 Courtiol P, Maussion C, Moarii M et al. Deep learning-based classification of mesothelioma improves prediction of patient outcome. Nature Medicine 2019;25:1519-25.

14 Spiga O, Cicaloni V, Fiorini C et al. Machine learning application for development of a data-driven predictive model able to investigate quality of life scores in a rare disease. Orphanet Journal of Rare Diseases 2020;15:46.

15 Basch E, Schrag D. The evolving uses of "real-world" data. JAMA 2019;321:1359-60.

16 Corrigan-Curay ], Sacks L, Woodcock J. Real-world evidence and real-world data for evaluating drug safety and effectiveness. JAMA 2018;320:867-8. 
17 Dranitsaris G, Zhu X, Adunlin G, Vincent MD. Cost effectiveness vs. affordability in the age of immuno-oncology cancer drugs. Expert Review of Pharmacoeconomics \& Outcomes Research 2018;18:351-7.

18 Topol E, Lee K-F. It takes a planet. Nat Biotechnol 2019;37:85861.
Address for correspondence: Dr Sanjay Budhdeo, Department for Clinical and Movement Neuroscience, National Hospital for Neurology and Neurosurgery, Queen Square, London, WC1B 3BG, UK.

Email: sanjay.budhdeo@doctors.org.uk Twitter: @DrSanjay_B 